\title{
A New Scheme for Calculation of the Multiplicity Distributions in Hadronic Interactions
}

\author{
P. C. Beggio a and Y. Hama ${ }^{\mathrm{b}}$ \\ ${ }^{a}$ Laboratório de Ciências Matemáticas, Universidade Estadual do Norte Fluminense Darcy Ribeiro, Campos dos Goytacazes - RJ, Brazil \\ ${ }^{\mathrm{b}}$ Instituto de Física, Universidade de São Paulo, C.P. 66318, 05315-970, São Paulo - SP, Brazil
}

Received on 23 July, 2007

\begin{abstract}
We generalize an existing geometrical approach for multiparticle production in hadronic interactions, in which the overall multiplicity distribution is given by summing contributions coming from each impact parameter $\mathbf{b}$ of the incident hadronic system. In the previous work, the process occurring at a fixed impact parameter $\mathbf{b}$ was interpreted as due to an elementary collision with formation of an object similar to the one in $e^{-} e^{+}$annihilations. Here, we extend the model allowing the possibility of formation of more than just one string. The output seems to be more consistent with data.
\end{abstract}

Keywords: Hadronic multiplicity distribution; Eikonal approximation; $p p$ and $\bar{p} p$ collisions

\section{INTRODUCTION}

Due to the lack of a computable fundamental theory, to describe the experimental data on multiplicity distributions, our strategy to investigate multiparticle production in hadronic collisions is still phenomenological and based on a wide class of models and some theoretical principles. Measurements of charged particle multiplicity distributions have revealed intrinsic features in hadronic interactions and provide important constraints on such models and can also indicate some suitable calculational schemes for further theoretical developments in QCD. In this work, we generalize an existing phenomenological procedure, referred to as geometrical approach, where the incident hadrons are treated as spatially extended objects and their collisions depicted as an ensemble of elementary interactions, characterized by the impact-parameter dependent available energy for producing particles. This approach allows simultaneous description of several experimental data from elastic and inelastic channels, once the inelastic overlap function is given and some parametrization both of the number of particles produced in elementary interactions and their distribution are assumed.

Observing that in $e^{-} e^{+}$annihilation probably one $q \bar{q}$ pair has triggered the multitude of final particles and this might be happening also in the above mentioned elementary interaction in hadronic collisions (either $q \bar{q}$ or $q(q q)$ string in this case), it has been assumed that the average multiplicity of the latter is identical to the former and its energy dependence given by the experimental data on $e^{-} e^{+}$annihilation and parametrized in a general power law form $\sim s^{A}$ ( $s$ is the center-of-mass energy squared). The idea of string formation for multiparticle production is similar to Lund model [1] and it has been applied also for hadron-hadron collisions by Rio de Janeiro group [2]. Two fit values of $A=0.258$ and $A=0.198$ have been obtained in the previous study [3], the first one giving a better account of lower energy data whereas the second one higher energy data. However, when the formalism is applied to multiplicity distribution in $\sqrt{s}=546 \mathrm{GeV} \bar{p} p$ collisions, the latter, which was expected to be more appropriate for this case, yielded a too narrow distribution, whereas the former, which overestimate the multiplicity in high energy region, gave a better account of the existing data [4]. This result may indicate that in actual hadron-hadron collisions the probability of creating two (or more) $q \bar{q}$ strings, besides the formation of just one $q \bar{q}$ pair, giving a larger multiplicity, is important. This is also physically more reasonable, since hadrons are composite objects.

The main object of the present paper is to show a new scheme of calculation for the overall multiplicity distributions in hadronic collisions, based on multistring formation. We shall begin, for completeness, by presenting the basic idea of geometrical approach in the next Section. In Sec. 3, we discuss an extension to multi-string models, giving two alternative treatments, namely, independent-string model and fusedstring model. Discussions of the new results and concluding remarks are given in Sec. 4.

\section{GEOMETRICAL APPROACH}

The elastic and diffractive scatterings are intimately related to the soft hadronic interaction (low $p_{T}$ ) and the differential cross section is an important physical observable in the elastic channel. From this, other quantities may be obtained, such as the integrated elastic cross section $\sigma_{e l}$ and the total cross section $\sigma_{t o t}$, through the unitarity condition. In counterpart, in the inelastic channel, the charged multiplicity distribution may be used to test specific models on hadronization mechanisms. In this work we would like to investigate the effects, on the overall multiplicity distributions, of the formation of two (or more) strings originated by $q \bar{q}$ (or $q(q q)$ ) pairs during the hadronization process in $p p$ and $p \bar{p}$ collisions. For this purpose we will begin discussing the basic ideas associated with the geometrical approach.

\section{A. Basic Formalism}

A geometrical approach has been discussed in reference [3] and, for completeness and especially to define the notation, we shall briefly review the main points here. In this approach hadrons are drawn as composite objects with some internal structure and the impact parameter formalism is used. By using the eikonal approximation, the connection between the 
elastic and the inelastic channels is established through the unitarity condition. Thus, it is possible to obtain simultaneous descriptions of some experimental data such as the differential elastic cross section, the inelastic cross section and multiplicity distribution. Specifically, the inelastic overlap function $G_{\text {in }}$ is related with the eikonal $\Omega$ by

$$
G_{i n}(s, b)=1-e^{-2 \Omega(s, b)},
$$

where $b$ is the impact parameter and $\sqrt{s}$ the center-of-mass energy. The inelastic cross section, $\sigma_{i n}(s)$, can be obtained directly from $G_{i n}(s, b)$ as

$$
\sigma_{\text {in }}(s)=\int d^{2} b G_{i n}(s, b) .
$$

Neglecting the real part of the amplitude and spin effects, the $s$-channel unitarity relation gives the differential elastic cross section, $d \sigma_{e l} / d t$, as [5]

$$
\frac{d \sigma_{e l}}{d t}=\left|\sqrt{\pi} \int_{0}^{\infty}\left[1-e^{-\Omega(s, b)}\right] J_{0}(b \sqrt{-t}) b d b\right|^{2} .
$$

In this work, the physical observable of interest is the overall (charged) multiplicity distribution and, in the impact parameter formalism, this may be constructed by summing contributions coming from hadron-hadron collisions taking place at fixed impact parameter $b$. In this way, the idea of a normalized multiplicity distribution at each impact parameter $b$ is introduced [6]. Thus the overall multiplicity distribution is written as

$$
P_{n}(s)=\frac{\sigma_{n}(s)}{\sigma_{i n}(s)}=\frac{\int d^{2} b G_{i n}(s, b)\left[\frac{\sigma_{n}(s, b)}{\sigma_{i n}(s, b)}\right]}{\int d^{2} b G_{i n}(s, b)},
$$

where the topological cross section $\sigma_{n}$ is decomposed into contributions from each impact parameter $b$ with weight $G_{i n}(s, b)$. We recall that the quantity in brackets can be interpreted as the probability of producing $n$ particles at impact parameter $b$ and that it should scale in KNO sense due to its elementary structure [3]. Then, in general,

$$
P_{n}(s)=\frac{\int d^{2} b \frac{G_{i n}(s, b)}{<n(s, b)>}\left[<n(s, b)>\frac{\sigma_{n}(s, b)}{\sigma_{\text {in }}(s, b)}\right]}{\int d^{2} b G_{i n}(s, b)},
$$

where $\langle n(s, b)\rangle$ is the average number of particles produced at $b$ and $\sqrt{s}$ due to the interactions among hadronic constituents involved in the collision. Let us write this quantity as [3]

$$
<n(s, b)>=<N(s)>f(s, b),
$$

where $\langle N(s)\rangle$ is the average multiplicity at $\sqrt{s}$ and call $f(s, b)$ multiplicity function. Now, similarly to $\mathrm{KNO}$, let us introduce for each $b$ the elementary multiplicity distribution

$$
\psi^{(1)}\left(\frac{n}{<n(s, b)>}\right)=<n(s, b)>\frac{\sigma_{n}(s, b)}{\sigma_{i n}(s, b)} .
$$

Thus, with Eqs. (6) and (7), Eq. (5) becomes

$$
\Phi(s, z)=\frac{\int d^{2} b \frac{G_{i n}(s, b)}{f(s, b)} \psi^{(1)}\left(\frac{z}{f(s, b)}\right)}{\int d^{2} b G_{i n}(s, b)}
$$

where $\Phi(s, z)=<N(s)>P_{n}(s)$, and $z=n /<N(s)>$ represents the usual KNO scaling variable.

Now to obtain the multiplicity function $f(s, b)$ in terms of the eikonal $\Omega$, we assume that

1. the fractional energy $\sqrt{s^{\prime}}$ that is deposited for particle production in a collision at $b$ is proportional to $\Omega$, which is usually understood as the target thickness,

$$
\sqrt{s^{\prime}}=\beta \Omega(s, b)
$$

2. in hadron-hadron collisions at $b$, the average number of produced particles depends on the energy $\sqrt{s^{\prime}}$ in the same way as in $e^{-} e^{+}$annihilations, which is approximately represented by a power law in $\sqrt{s}$ [3] (This behavior can arise, for instance, from a simple picture of branching decay producing a tree structure [7].)

$$
<n(s, b)>=\gamma s^{\prime} A .
$$

Combining Eqs. (9), (10) and (6), we have

$$
f(s, b)=\xi(s)[\Omega(s, b)]^{2 A},
$$

with $\xi(s)$ determined by the usual normalization conditions on $\Phi$,

$$
\int_{0}^{\infty} \Phi(s, z) d z=2=\int_{0}^{\infty} \Phi(s, z) z d z
$$

The first of these conditions is satisfied, if $\psi^{(1)}$ also obeys the same normalization condition. As for the second one, however, if $\psi^{(1)}$ obeys the same condition, it serves to determine $\xi(s)$ as an energy dependent quantity, giving

$$
\xi(s)=\frac{\int d^{2} b G_{i n}(s, b)}{\int d^{2} b G_{i n}(s, b)[\Omega(s, b)]^{2 A}} .
$$

Adopting an appropriate parametrization for $G_{i n}, \psi^{(1)}$ and an adequate value for $A$, we can test the formalism embodied in Eq. (8), with Eqs. (11) and (13), making direct comparisons with overall multiplicity-distribution data. In the following, we will shortly discuss the results obtained in Ref. [3], before going to the next Section where this approach will be generalized.

\section{B. Simple One-String Model: Inputs and Results}

The formalism of geometrical approach is based on the idea that in hadron-hadron collisions, taking place at a fixed value of $b$, particles are produced due to the elementary interaction between quarks and/or gluons. As pointed out in [8], what the elementary hadronic processes are depends on the model 
used, and, what the elementary multiplicity distribution $\psi_{1}$ should be also varies from model to model. On these arguments and following other authors [6], Beggio, Menon and Valin [3] considered the experimental data available on $e^{-} e^{+}$ annihilations as possible and limited source of information concerning elementary hadronic interactions, without looking for connections between $e^{-} e^{+}$annihilations and $p(\bar{p})-p$ collisions. Thus, assuming a gamma distribution normalized to 2 ,

$$
\psi^{(1)}(z)=2 \frac{k^{k}}{\Gamma(k)} z^{k-1} e^{-k z},
$$

for $e^{-} e^{+}$multiplicity distributions, experimental data covering the interval $22.0 \mathrm{GeV} \leq \sqrt{s} \leq 161 \mathrm{GeV}$ were fitted, obtaining $\mathrm{k}=10.775 \pm 0.064\left(\chi^{2} / N_{D F}=2.61\right)$. Also, the average multiplicity data in $e^{-} e^{+}$annihilations, were fitted by Eq.(10), giving $A=0.258 \pm 0.001\left(\chi^{2} / N_{D F}=8.89\right)$ in the interval $5.1 \mathrm{GeV} \leq \sqrt{s} \leq 183 \mathrm{GeV}$, and $A=0.198 \pm 0.004$ $\left(\chi^{2} / N_{D F}=1.7\right)$ for the set in the interval $10 \mathrm{GeV}<\sqrt{s} \leq 183$ $\mathrm{GeV}$, respectively. For the sake of discussions presented in the next Section, we have collected, in Fig. 1, the average multiplicity data in the interval $10 \mathrm{GeV}<\sqrt{s} \leq 200 \mathrm{GeV}$ [9] and, compared with a new fit to Eq. (10), with $\gamma=3.36$ and $A=0.200$ with $\left(\chi^{2} / N_{D F}=0.94\right)$. The $A$ parameter is essencially the same of the one of those used in Ref. [3].

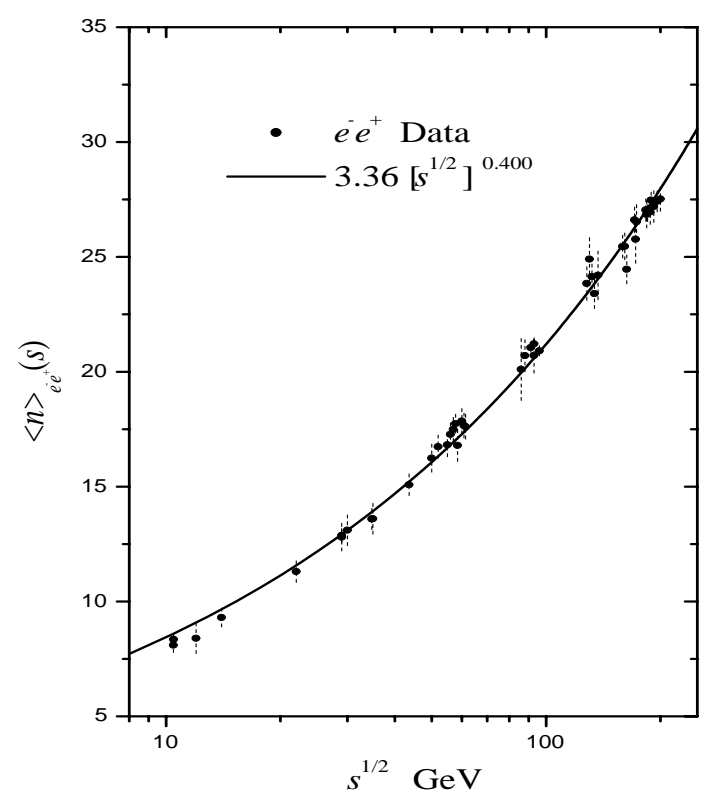

FIG. 1: Average charged multiplicity data for $e^{-} e^{+}$annihilation [9] and the fitted power law, Eq. (10).

Adopting the Henzi - Valin parametrization [10] for $G_{i n}(s, b)$, which describes well the energy dependence of the differential elastic distributions, the authors of Ref. [3] concluded that the energy evolution of the overall hadronic multiplicity distributions, from ISR to Collider $(30 \sim 500 \mathrm{GeV})$, is correctly reproduced without changing the underlying elementary interaction, in agreement with what could be expected from QCD [3].

As mentioned above, the Geometrical Approach is based on the idea of multiparticle creation due to the interactions between hadronic constituents in collisions taking place at $b$. It is at this point that specific models for the hadronization mechanisms could be introduced. However, we shall keep the previous assumption that the $e^{-} e^{+}$annihilation data are a possible source of information concerning the elementary hadronic interactions. In fact, Basile et al. [11] found that the average multiplicity in $p p$ collisions is similar to that for $e^{-} e^{+}$collisions. It was concluded that the multiparticle production mechanism is the same in both processes, controlled mainly by the amount of energy available for particle production. It is important, for our purpose in this paper, to look at some aspects of physical scenario associated with hadron production both in $e^{-} e^{+}$annihilations and $p p$ collisions. In $e^{-} e^{+}$collisions, they are annihilated into a virtual photon or $Z$, which decays into a $q \bar{q}$ pair. The primary quarks radiate gluons, which can radiate further gluons or be converted into $q \bar{q}$ pairs giving rise to a parton cascade. Subsequently the partons hadronize and the unstable hadrons decay [12] ( $e^{-} e^{+} \longrightarrow Z /(\gamma) \longrightarrow q \bar{q} \longrightarrow$ hadron). To implement our analysis let us call a $q \bar{q}$ pair creation as "one chain" formation. In the previous work, two values for the exponent in Eq. (10) were obtained, namely, $A=0.258$ and 0.198. As shown there, indeed smaller value of $A$ gives a narrower overall multiplicity distribution in hadronic collisions as compared to the one with larger $A$. However, despite a better agreement with the overall multiplicity distribution data at $\sqrt{s}=546 \mathrm{GeV}$ that $A=0.258$ gave, we should prefer $A=0.198$, that better fits the $e^{-} e^{+}$average multiplicity data at high-energy domain, to be consistent with our basic assumption that $e^{-} e^{+}$annihilations are a possible source of information on hadronization mechanism. The fact that $A=0.258$, which enhances larger multiplicities, gave a better account of $p \bar{p}$ multiplicity distribution data may indicate that, instead of one chain with larger multiplicity, two or more chains are being formed. This is also a physically more realistic description of interaction between compound objects such as hadrons, because we expect that more than one elementary collisions tend to occur in smaller $b$ collisions, where the overlap of hadronic matter is larger and hence two or more $q \bar{q}$ pairs, or equivalently two or more chains could be produced. What are the effects of formation of two or more chains in hadronic interactions? In the next Section we shall generalize the framework presented above, to include the contributions of various chains in multiparticle production.

\section{GENERALIZATION TO MODELS WITH MULTIPLE STRINGS}

In this Section, we shall modify the original geometrical approach, on the assumption that there may occur formation of several chains in hadronic interactions. Let us start by ex- 
panding $G_{\text {in }}(s, b)$, given by Eq. (1), in the following form

$$
\begin{aligned}
G_{i n}(s, b) & =\sum_{i=1}^{\infty} \frac{[2 \Omega(s, b)]^{i}}{i !} e^{-2 \Omega(s, b)} \\
& \equiv \sum_{i=1}^{\infty} G^{(i)}(s, b) .
\end{aligned}
$$

Since $2 \Omega(s, b)$ could be interpretted as the "thickness" of interacting hadrons at $b$ and $\sqrt{s}$, we expect that, in eikonal approximation, the probability of forming $i$ chains in a collision at $b$ and $\sqrt{s}$ is some thing proportional to the $i$-th term of the expansion above (15). Let us call it overlap function for formation of $i$ chains, $G^{(i)}(s, b)$.

By integrating Eq. (16), term by term, we obtain the total inelastic cross section as a sum of partial cross sections for formation of multiple number of chains

$$
\begin{aligned}
\int d^{2} b G_{i n}(s, b) & =\sum_{i=1}^{\infty} \int d^{2} b G^{(i)}(s, b) \\
\Rightarrow \sigma_{i n}(s) & =\sigma_{i n}^{(1)}(s)+\sigma_{i n}^{(2)}(s)+\sigma_{i n}^{(3)}(s)+\ldots
\end{aligned}
$$

Now, dividing this by $\sigma_{i n}(s)$, we can define the $i$-chain formation probability, $q^{(i)}(s)$, as

$$
q^{(i)}(s)=\frac{\sigma_{i n}^{(i)}(s)}{\sigma_{i n}(s)}=\frac{\int d^{2} b G^{(i)}(s, b)}{\int d^{2} b G_{i n}(s, b)} .
$$

Remark that, in view of Eq. (17), the correct normalization condition

$$
\sum_{i=1}^{\infty} q^{(i)}(s)=1
$$

is automatically satisfied.

Based on this new formalism, we can obtain all the elements for the calculation of the overall multiplicity distributions, considering formation of various chains in the interactions among hadronic constituents. In the following subsections we will consider two possible schemes for this purpose.

\section{A. Independent-String Model}

First, let us suppose our $i$ strings introduced above decay independently. Then, the overall multiplicity distribution is a sum of the multiplicity distributions associated with each set of $i$ chains, $\phi^{(i)}$

$$
\Phi(s, z)=\sum_{i=1}^{\infty} \phi^{(i)}(s, z)
$$

with

$$
\phi^{(i)}(s, z)=\frac{\int d^{2} b \frac{G^{(i)}(s, b)}{f^{(i)}(s, b)} \psi^{(i)}\left(\frac{z}{f^{(i)}(s, b)}\right)}{\int d^{2} b G_{i n}(s, b)} .
$$

Here, $\psi^{(i)}$ represents the elementary multiplicity distribution at the impact parameter $b$ and, now, the weight function is the inelastic overlap function associated with $i$ chains and $f^{(i)}(s, b)$ the multiplicity function of $i$ chains. As for the latter, remark that the average multiplicity depends now on the number of chains produced in the collision. Thus, let us write the average multiplicity associated with each $i$ chains as

$$
<n(s, b)>^{(i)}=i \gamma s^{\prime} A .
$$

By analogy with Eq. (6), this is related to the multiplicity function of $i$ chains as

$$
<n(s, b)>^{(i)}=<N(s)>f^{(i)}(s, b) .
$$

Recalling that the fractional energy deposited at $b$ for particle production is proportional to $\Omega$, as expressed by Eq. (9), the multiplicity function of $i$ chains is written

$$
f^{(i)}(s, b)=i \xi(s)[\Omega(s, b)]^{2 A} .
$$

By integrating $\Phi(s, z)$, given by Eq.(20), and using Eqs. $(18,19)$, we obtain

$$
\int_{0}^{\infty} \Phi(s, z) d z=2 \sum_{i=1}^{\infty} q^{(i)}(s)=2,
$$

so it correctly obeys the normalization condition, Eq.(12). We can also verify that $\xi$ is given as

$$
\xi(s)=\frac{\int d^{2} b G_{i n}(s, b)}{\int d^{2} b[\Omega(s, b)]^{2 A}\left[\sum_{i=1}^{\infty} i G^{(i)}(s, b)\right]} .
$$

Now, let us consider how to specify $\psi^{(i)}$ in Eq. (21). Confining ourselves just to two independent-string formation, we need to specify $\psi^{(1)}$ and $\psi^{(2)}$. On the assumption that the multiplicity distribution of each chain is independent and have the same form of $\psi^{(1)}$, we may write $\psi^{(2)}$ as a convolution product

$$
\psi^{(2)}(z)=2 \int_{0}^{z}\left[\frac{1}{2} \psi^{(1)}\left(z^{\prime}\right)\right]\left[\frac{1}{2} \psi^{(1)}\left(z-z^{\prime}\right)\right] d z^{\prime},
$$

where the factors 2 and $\frac{1}{2}$ are due to the normalization condition. With $\psi^{(1)}$ being given by Eq. (14), we obtain

$$
\psi^{(2)}(z)=2 \frac{(2 k)^{2 k}}{\Gamma(2 k)} z^{2 k-1} \exp (-2 k z),
$$

which can be verified that is correctly normalized as

$$
\int_{0}^{\infty} \psi^{(2)}(z) d z=2=\int_{0}^{\infty} \psi^{(2)}(z) z d z
$$

For the general case of $i$ independents chains, we have

$$
\psi^{(i)}(z)=2 \frac{(i k)^{i k}}{\Gamma(i k)} z^{i k-1} \exp (-i k z)
$$

With the results above we have all the necessary elements to test the formalism embodied in Eqs. (20) and (21). For instance, if we limit ourselves only to one or two independentchain formation, the overall multiplicity distribution is explicitly given by

$$
\begin{aligned}
\Phi(s, z) & =\frac{\int d^{2} b \frac{G^{(1)}(s, b)}{\xi(s)[\Omega(s, b)]^{2 A}} \psi^{(1)}\left(\frac{z}{\xi(s)[\Omega(s, b)]^{2 A}}\right)}{\int d^{2} b G_{i n}(s, b)} \\
& +\frac{\int d^{2} b \frac{G^{(2)}(s, b)}{2 \xi(s)[\Omega(s, b)]^{2 A}} \psi^{(2)}\left(\frac{z}{2 \xi(s)[\Omega(s, b)]^{2 A}}\right)}{\int d^{2} b G_{i n}(s, b)} .
\end{aligned}
$$




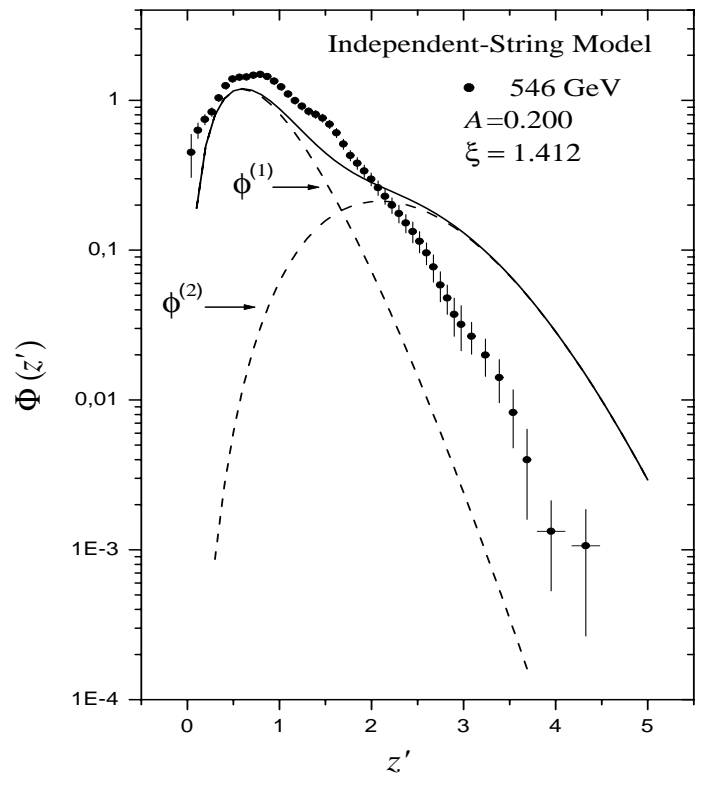

FIG. 2: Scaled multiplicity distribution data for inelastic $p \bar{p}$ at $\sqrt{s}=546 \mathrm{GeV}$ [4] compared to theoretical prediction using the Independent-String Model, Eqs. (20) and (21) (solid-line). $\phi^{(i)}(i=$ $1,2)$ (dashed lines) are the multiplicity distributions for one and two chains given by Eq. (21).

We show, in Figure 2, the result of computation of this $\Phi$ at the CERN $\bar{p} p$-Collider energy $(\sqrt{s}=546 \mathrm{GeV})$ together with the experimental data [4]. Here we fixed the value of $A=0.2$ as obtained in Section 2, $G_{\text {in }}$ is that obtained by Henzi and Valin [10], $G^{(i)}(s, b)(i=1,2)$ given by Eq. $(16), \psi^{(1)}$ and $\psi^{(2)}$ by Eqs. (14) and (28) and the value of $\xi(s)$ was obtained with Eq. (26). We also expressed $\Phi$ in terms of the modified scaling variable $z^{\prime}=n-N_{o} /\left\langle n-N_{o}\right\rangle$ with $N_{o}$ representing the average number of leading particles, an essentially energy independent quantity whose value is $0.9[3,13]$. One can clearly see the disagreement of the theoretical curve when compared to the data in the entire $z^{\prime}$ interval. It is broader than $\phi^{(1)}$ as we would like, but too broad.

We would like to observe that others forms could be used as imput to Eikonal function $\Omega$, however, the overall multiplicity distributions, in the geometrical approach, are stable against variations on the details about $\Omega$ [3].

\section{B. Fused-String Model}

Now, let us formulate a modified version of the preceding model, in which the constituents of the incident hadrons interact at certain $b$, initially forming $i$ chains ( $i q \bar{q}$ pairs). However, since they are formed in a very small region of typical hadron size, it is natural that they are subsequently fused to from just one larger chain. We make the simplest assumption that this final chain decays producing particles with distribution $\psi^{(1)}$ given by Eq. (14), as in the simple one-string model.
We shall still assume that the average energy of each initial chain is equal and given by Eq. (9), so that the total energy $\sqrt{s^{\prime}}$ of a fused chain, originated from $i$ initial chains, is written

$$
\sqrt{s^{\prime}}=i \beta \Omega(s, b),
$$

with $i$ representing the number of initial chains created in the interaction.

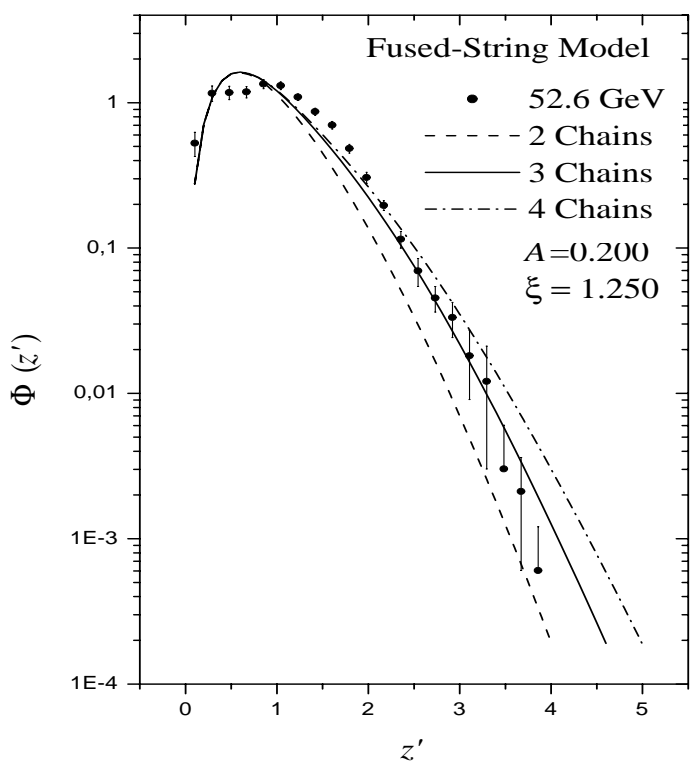

FIG. 3: Overall scaled multiplicity distribution data for inelastic $p p$ at ISR energy [14], compared to theoretical prediction using the Fused-String Model, Eqs. (35) and (36).

As for the average multiplicity of the fused chain, we assume it depends on the energy $\sqrt{s^{\prime}}$ in the same way as the one expressed by Eq. (10). Thus, to adequate the notation, let us rewrite the average multiplicity originated from $i$ initial chains as

$$
<n(s, b)>^{(i)}=\gamma s^{\prime} A,
$$

and the multiplicity function has the same form as Eq. (23). Combining the Eqs. (32), (33) and (23) we obtain

$$
f^{(i)}(s, b)=i^{2 A} \xi(s)[\Omega(s, b)]^{2 A} .
$$

In this model the overall multiplicity distribution $\Phi(s, z)$ is still given by Eq. (20) but, due to the fusion of the $i$ chains into a final chain, the multiplicity distribution $\phi^{(i)}(s, z)$ associated with this fused chain is written as

$$
\phi^{(i)}(s, z)=\frac{\int d^{2} b \frac{G^{(i)}(s, b)}{f^{(i)}(s, b)}\left[\psi^{(1)}\left(\frac{z}{f^{(i)}(s, b)}\right)\right]}{\int d^{2} b G_{i n}(s, b)} .
$$


Naturally $\Phi$ obtained in the fused-string model, given by Eqs. (20) and (35), must obeys the usual normalization condition expressed by Eq. (12), which gives $\xi$ as

$$
\xi(s)=\frac{\int d^{2} b G_{i n}(s, b)}{\int d^{2} b[\Omega(s, b)]^{2 A}\left[\sum_{i=1}^{\infty} i^{2 A} G^{(i)}(s, b)\right]} .
$$

By fixing the value of $A=0.2$ as before, adopting $G_{\text {in }}$ from analysis by Henzi and Valin as discussed in the last subsection and observing that $\xi(s)$ is now obtained by Eq. (36), we have computed the overall multiplicity distribution at ISR and Collider energies. The theoretical curve at $\sqrt{s}=52.6 \mathrm{GeV}$ is shown in Fig. 3 together with the experimental data [14] and we can see that the curve with formation of up to three initial chains shows good agreement with the data. Comparison at Collider energy is shown in Fig. 4. It is seen that a better agreement with data is obtained, if we assume formation of up to four initial chains in this case.

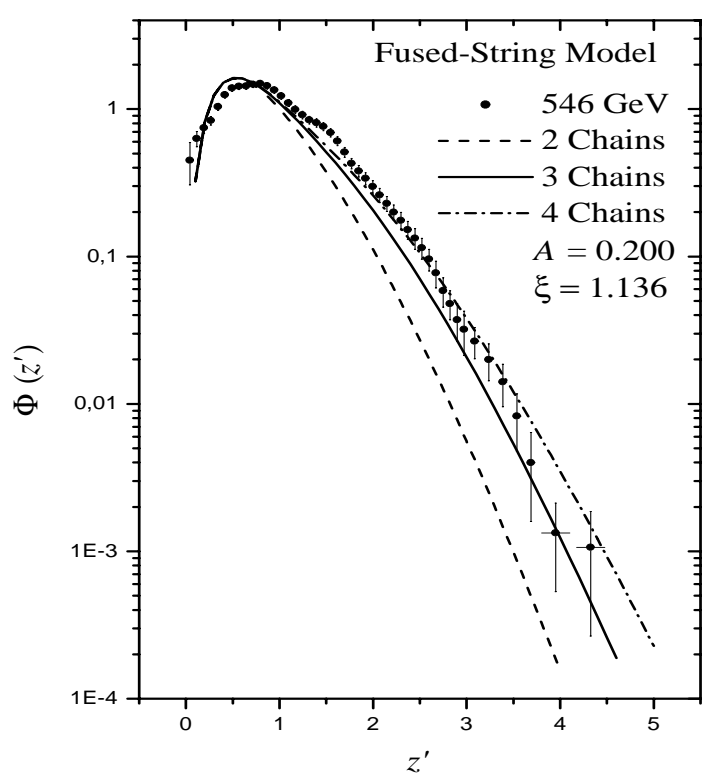

FIG. 4: Overall scaled multiplicity distribution for inelastic $p \bar{p}$ data at Collider energy [4] compared to theoretical prediction using the Fused-String Model, Eqs. (35) and (36).

\section{DISCUSSION AND CONCLUDING REMARKS}

In the preceding Section, we have generalized an existing geometrical approach for multiplicity distributions in hadronhadron inelastic interaction [3], allowing the production of two or more $q \bar{q}$ (or $q(q q)$ ) strings in the interaction. By modifying the previously proposed Simple One-String Model, we have formulated two alternative models: $i$ ) IndependentString Model, where the produced multiple chains decay independently; and ii) Fused-String Model, where the multiple initial chains are fused into one before the decay into final produced particles. We have shown that although both of the models do predict the broadenning of the distribution as expected, the curves from Independent-String Model become too broad, already with two chains, revealing clear discrepancy with all the data at Collider energies, as shown in Fig. 2. As for the Fused-String Model, the predictions compare better with the data, giving reasonable agreement with up to three chains at $\sqrt{s}=52.6 \mathrm{GeV}$ (Fig. 3), and with up to four chains at $\sqrt{s}=546 \mathrm{GeV}$ (Fig. 4), so this version should be preferred over the first one. This conclusion is also reasonable from the physical point of view, because since the $q \bar{q}$ pair formation occurs in a rather small volume, it is natural that they evolve into just one large chain instead of remaining many independent chains.

The increase in the number of the initially formed strings to fit the data as the incident energy increases is also natural, since the parton number increases with increasing the incident energy. In principle, according to Eqs. (16) and (20), the models described in the preceding Section should contain infinite terms, corresponding to infinite number of chains, irrespective of the incident energy. However, evidently there are physical restrictions, such as the finite available energy and the finite effective partons in the incident hadrons, which forbid to form indefinitely large number of strings.

\section{Acknowledgements}

This work has partially supported by FAPESP (Fundação de Amparo à Pesquisa do Estado de São Paulo) under the contract numbers 2000/04422-7 and 2004/10619-9.
[1] X. Artru and G. Mennesier, Nucl. Phys. B70, 93 (1971); B. Anderson, G. Gustafson, G. Ingelman, T. Sjöstrand, and X. Artru, Phys. Rep. 97, 31 (1983).

[2] R. A. M. S. Nazareth, N. Prado, and T. Kodama, Phys. Rev. D40, 2861 (1989); R. A. M. S. Nazareth, T. Kodama, and D. A. Portes, Jr., Phys. Rev. D46, 2896 (1992).

[3] P. C. Beggio, M. J. Menon, and P. Valin, Phys. Rev. D61, 034015 (2000)

[4] UA5 Collaboration, G. J. Alner et al., Phys. Rep. 154, 247 (1987).

[5] T. T. Chou and C. N. Yang, Phys. Rev. 170, 1591 (1968); R.
Henzi and P. Valin, Phys. Lett. B 48, 119 (1974).

[6] S. Barshay, Phys. Rev. Lett. 49, 1609 (1982).

[7] S. Rudaz and P. Valin, Phys. Rev. D34, 2025 (1986).

[8] C. S. Lam and P. S. Yeung, Phys. Lett. B 119, 445 (1982).

[9] OPAL Collaboration: P. D. Acton et al., Z. Phys. C53, 539 (1992) and references therein; R. Akers et al., Z. Phys. C68, 203 (1995); G. Alexander et al., Z. Phys. C72, 191 (1996); K. Ackerstaff et al., Z. Phys. C75, 193 (1997); G. Abbiendi et al., Eur. Phys. J. C16, 185 (2000);

ALEPH Collaboration: D. Buskulic et al., Z. Phys. C69, 15 (1996); Z. Phys. C73, 409 (1997); 
DELPHI Collaboration: P. Abreu et al., Phys. Lett. B372, 172 (1996); Phys. Lett. B416, 233 (1998); Eur. Phys. J. C6, 19 (1999); Eur. Phys. J. C18, 203 (2000);

L3 Collaboration: M. Acciarri et al., Phys. Lett. B371, 137 (1996); Phys. Lett. B404, 390 (1997); Phys. Lett. B444, 569 (1998);

TOPAZ Collaboration: K. Nakabayashi et al., Phys. Lett. B413, 447 (1997);

VENUS Collaboration: K. Okabe et al., Phys. Lett. B423, 407 (1998);

ARGUS Collaboration: H. Albrecht et al., Z. Phys. C54, 13
(1992).

[10] R. Henzi and P. Valin; Phys. Lett. B 160, 167 (1985).

[11] M. Basile et al., Phys. Lett. B 92, 367 (1980).

[12] A. de Angelis, in Procedings of the Fourth Gleb Wataghin School on High Energy Phenomenology, July 8-12, (1996) Unicamp, Campinas, SP, Brazil.

[13] P. Valin; Z. Physik C34, 313 (1987).

[14] ABCDWH Collaboration; A. Breakstone et al., Phys. Rev. D30, 528 (1984). 